\title{
Epidemiological Data, Serovar Distribution and Antimicrobial Resistance Patterns of Salmonella Species in Children, Greece 2011-2017: A Retrospective Study
}

\author{
Grigoris Grivas $^{1}$, Theano Lagousi ${ }^{2,3}$, Georgia Mandilara ${ }^{4}$ \\ ${ }^{1}$ Penteli Children's Hospital, Pediatric Intensive Care Unit, Athens, Greece, ${ }^{2}$ Infectious Diseases Unit and University Research \\ Institute for the Study of Genetic \& Malignant Disorders in Childhood, 1st Department of Paediatrics, Aghia Sofia Children's \\ Hospital, National and Kapodistrian University of Athens, Athens, Greece, ${ }^{3}$ Penteli Children's Hospital, Department of \\ Pediatrics, Athens, Greece, ${ }^{4}$ School of Public Health, Faculty of Public Health Policies, University of West Attica, National \\ Reference Centre for Salmonella-Shigella, Athens, Greece
}

Correspondence: theanolagousi@hotmail.com; Tel.: + 3062132052 412, Fax.: + 302132052823

Received: 13 April 2020; Accepted: 12 October 2020

\begin{abstract}
Objective. This study aimed to describe Salmonella epidemiology and antimicrobial resistance in Greek children over the period of 2011-2017. Materials and Methods. A 7-year retrospective study (2011-2017) was performed, based on data recorded by the National Reference Centre for Salmonella, among children aged $\leq 14$ years. Epidemiological data, serovar distribution and antimicrobial resistance patterns were recorded. Results. Overall, 2347 Salmonella isolates were collected (27 typhoidparatyphoid). Salmonellosis cases increased by almost 2-fold in 2017 compared to 2011 . The highest rates were reported in August, with infants being the most vulnerable group (17.9\%). The majority of isolates were identified in stool samples (91\%). Boys slightly outnumbered girls ( 1.05:1). Salmonella Enteritidis was the most prevalent serovar (28.5\%), followed by Salmonella Typhimurium (12.2\%) and Salmonella monophasic Typhimurium (10.4\%). Non-typhoid isolates displayed low resistance rates to $3^{\text {rd }}$ generation cephalosporins $(1 \%)$ and ciprofloxacin $(0.3 \%)$, while the corresponding resistance of typhoid isolates was $10 \%$ and $5 \%$ respectively. An increasing trend of Salmonella monophasic Typhimurium was recorded, associated with high rates of multidrug resistance, reaching a percentage of $97.8 \%$ in 2017. Conclusions. Salmonellosis epidemiology in Greek children is comparable to previously published European data. Antimicrobial resistance rates to $3^{\text {rd }}$-generation cephalosporins and ciprofloxacin for non-typhoid and typhoid-paratyphoid remain low. Notably, there is an increasing prevalence of Salmonella monophasic Typhimurium isolates, associated with multiple antimicrobial resistance.
\end{abstract}

Key Words: Salmonella • Epidemiology • Antimicrobial Resistance $・$ Serovars $・$ Children.

\section{Introduction}

Salmonella infection (Salmonellosis) is a global public health problem, predominantly among children, associated with significant morbidity and mortality (1). Salmonella species (Salmonella spp.) cause typhoid fever and non-typhoid salmonellosis that causes gastroenteritis, rarely associated with complications, such as reactive arthritis and bacteremia.

Salmonella serotype Typhi and Salmonella serotype Paratyphi A, B, and C (ie, typhoidal Salmonella) are responsible for causing typhoid fever in humans, an illness that is most burdensome in the developing world. The World Health Organization (WHO) estimates 16 to 33 million cases of typhoid fever causing 500,000 to 600,000 deaths worldwide annually (2).

Non-typhoidal Salmonella (NTS) species, which are found widely in animals, are estimated to cause more than 90 million illnesses worldwide, and to account for approximately 155,000 deaths each year (2). The transmission of salmonellosis is frequently associated with the consumption of contaminated water and food of animal origin (i.e. eggs, meat, dairy products), and is facilitated by conditions characterized by poor hygiene (3). Young children and infants are at increased risk 
of acquiring salmonellosis, while children with certain underlying conditions (such as hemoglobin disorders, infection with human immunodeficiency virus (HIV), malignancy, or other causes of immune suppression) have an increased risk of severe disease and death from complications (3).

Salmonella monitoring and control programs have been recently implemented in the European Union (EU). In contrast to the earlier declining trend of human salmonellosis (4), there have been some unfavorable changes on this trend since 2016 in Europe (5). In self-limiting gastrointestinal infections, antimicrobial treatment is not required. On the other hand, antimicrobial treatment is essential and life-saving in cases of invasive salmonellosis, mainly among children, the elderly and the immunocompromised (6).

The increasing resistance rates against traditional agents (aminopenicillins, trimethoprim-sulfamethoxazole, and chloramphenicol) have caused a shift to fluoroquinolones and $3^{\text {rd }}$-generation cephalosporins in empirical treatment of salmonellosis $(7,8)$. Nevertheless, under selection pressure, resistance to fluoroquinolones and extended-spectrum $\beta$-lactamases (ESBL) has been reported, and multidrug resistant (MDR) Salmonella spp. isolates have emerged (9-11). Several factors contribute to reducing the efficiency of a targeted antimicrobial treatment, such as: subpopulations of Salmonella showing increased survival after exposure to antibiotics (12), the presence in the host of more than one strain with different antimicrobial susceptibility (13), the selection pressure and the possibility of transferring the resistance between bacteria through mobile genetic elements conferring resistance (14-16). Given the importance of salmonellosis in global foodborne illnesses and in childhood diarrhea, knowledge of local epidemiology and antibiotic resistance patterns is important to determine suitable therapeutic and control strategies.

This study aimed to describe the epidemiological data, serovar distribution and antimicrobial resistance of Salmonella spp. isolates among children aged $\leq 14$ years old in Greece, based on data recorded by the National Reference Centre for Salmonella (NRCS) over the period from 2011 to 2017.

\section{Materials and Methods}

All human salmonellosis cases diagnosed by clinical and diagnostic laboratories are mandatorily reported to the Hellenic Centre for Disease Control and Prevention (HCDCP); Salmonella isolates are voluntarily forwarded to the NRCS for serotyping and antimicrobial susceptibility testing. A 7-year retrospective study (2011 - 2017) was carried out, through the NRCS data, among children aged $\leq 14$ years old. When two or more specimens corresponded to the same caseinfectious episode, the latter was separately defined in the analysis to exclude duplicate surveillance data. Epidemiological data were recorded, while serotyping was performed according to the White-KaufmannLe Minor Scheme. Susceptibility testing was assessed using the disk diffusion method. Antimicrobial agents appropriate for monitoring the antibiotic resistance in human Salmonella isolates were tested, according to the relevant EU protocol (17). The antimicrobial agents were evaluated, including: ampicillin, amoxicillin-clavulanic acid, amikacin, ceftazidime, ciprofloxacin, chloramphenicol, cefotaxime, tobramycin, netilmicin, nalidixic acid, streptomycin, sulfamethoxazole (that was available only during the sub period of 2014 to 2017), tetracycline and trimethoprim. Multidrug resistance was defined as acquired non-susceptibility to at least one agent in three or more classes of antimicrobials. The EU Antimicrobial Susceptibility Testing breakpoints (2010) were applied. Isolates that displayed intermediate susceptibility to the antimicrobial agent being tested were classified as resistant, since even if they inhibit the bacterium in vitro, they are excluded from routine clinical practice. The Local Institute Ethics Review Board approved the study protocol (35/1625/2011).

\section{Statistical Analysis}

A descriptive statistical analysis was performed. Categorical variables (gender, age, specimen source, geographical data, antimicrobial resistance) are expressed as frequencies and percentages. Time trends in the occurrence of salmonellosis were assessed with time series analysis (ARIMA modeling procedure). Analyses were undertaken with the SPSS v. 18.0. $\mathrm{P}<0.05$ was considered the criterion of significance. 


\section{Results}

\section{Epidemiology}

In the 7-year period spanned by this study, NRCS received 2347 Salmonella spp. isolates from children aged $\leq 14$ years old (Figure 1A). Overall, 117 different serovars were identified. A similar trend was observed in the total number of salmonellosis cases reported during the study period, in all age groups (children and adults) (Figure 1A) (http://www.mednet. gr/whonet/). Salmonellosis cases observed in children aged $\leq 14$ years constantly represented a higher proportion of the total salmonellosis cases (Figure 1B).

Data on the date of collection were available for 2170 cases (92.5\%). The seasonal variation of salmonellosis is shown in Figure 2, while Figure 3 shows age distribution. Gender data were available for 2302 clinical isolates (98.1\%). Boys slightly outnumbered girls (overall ratio $\sim 1.05: 1$ ), and this difference remained fairly constant during the whole study period $(\mathrm{P}=0.44,>0.05)$. For all the cases included in our study, specimen source data were available. The majority of isolates were identified from stool samples (91\%), followed by blood (3\%) and urine (0.7\%). Geographical data were available for 2321 clinical isolates (98.9\%). The majority by year. Greece, 2011-2017. were recorded in Athens, where rates remained almost stable over the first 5 years of the study period, ranging from 173 to 215 , but significantly increased in the last two years, 2016 and 2017, (346 and 384 respectively) $(\mathrm{P}<0.05)$.

All the cases were acquired in Greece, with 1 possible exception of a child with enteric fever caused by Salmonella Typhi, who had history of recent travel to France.
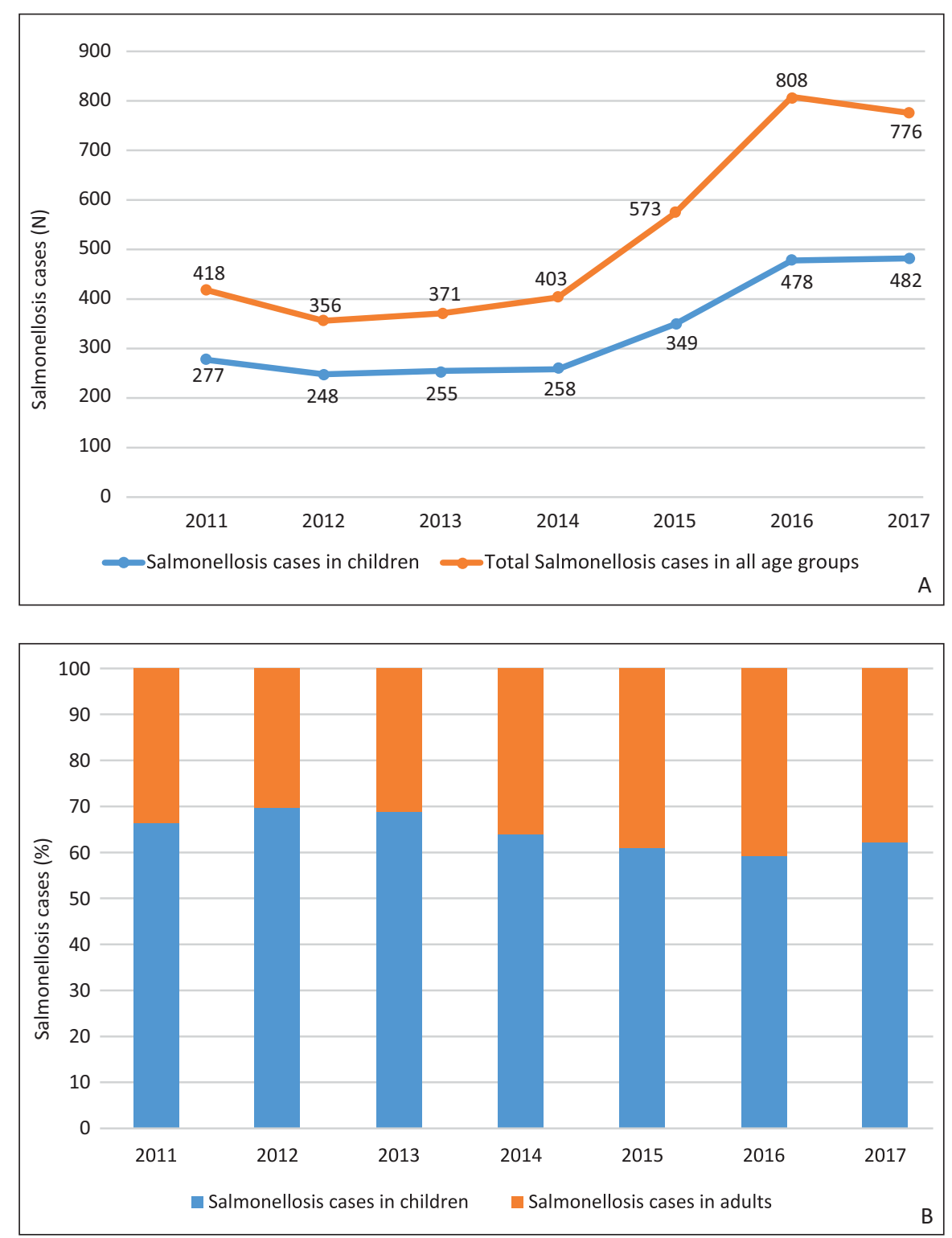

Figure 1. A: Annual rates of Salmonellosis cases in children $\leq 14$ years old, Greece, 2011 2017; B: Percentage distribution of Salmonellosis cases in children $\leq 14$ years old and adults 


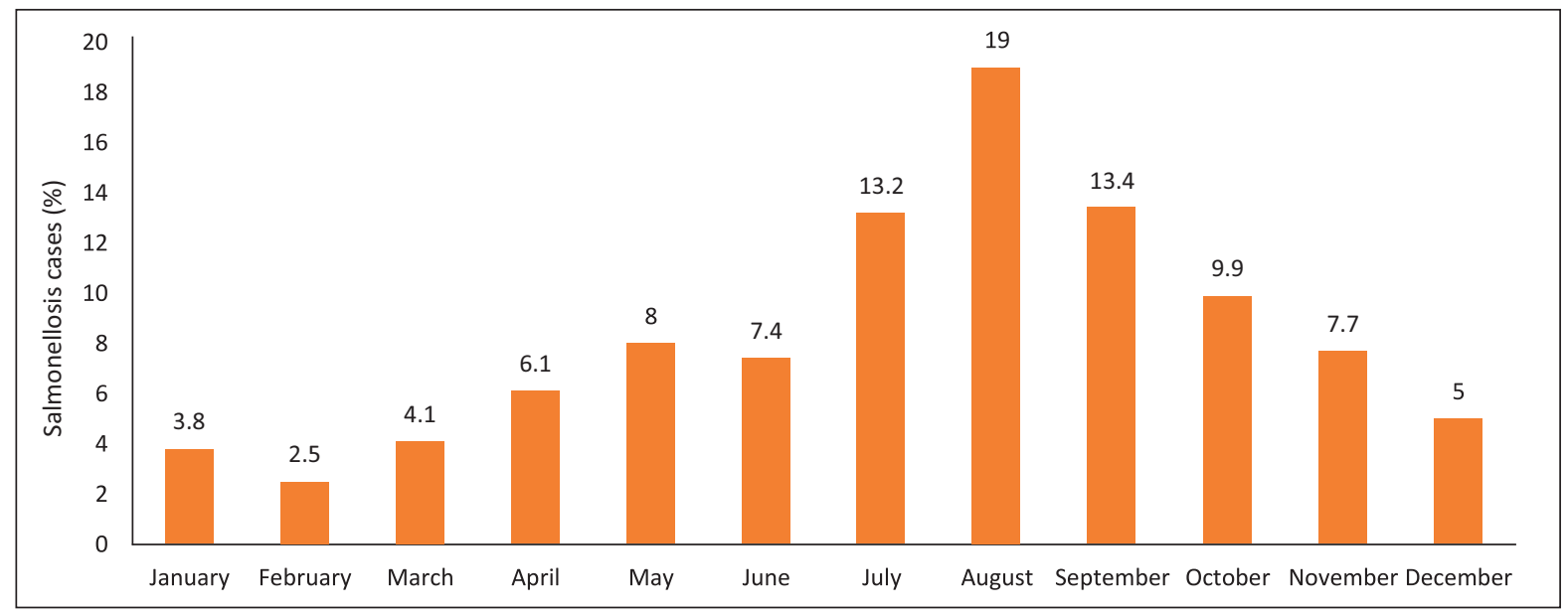

Figure 2. Seasonal percentage distribution of Salmonellosis cases in children aged $\leq 14$ years old, Greece, 2011-2017.

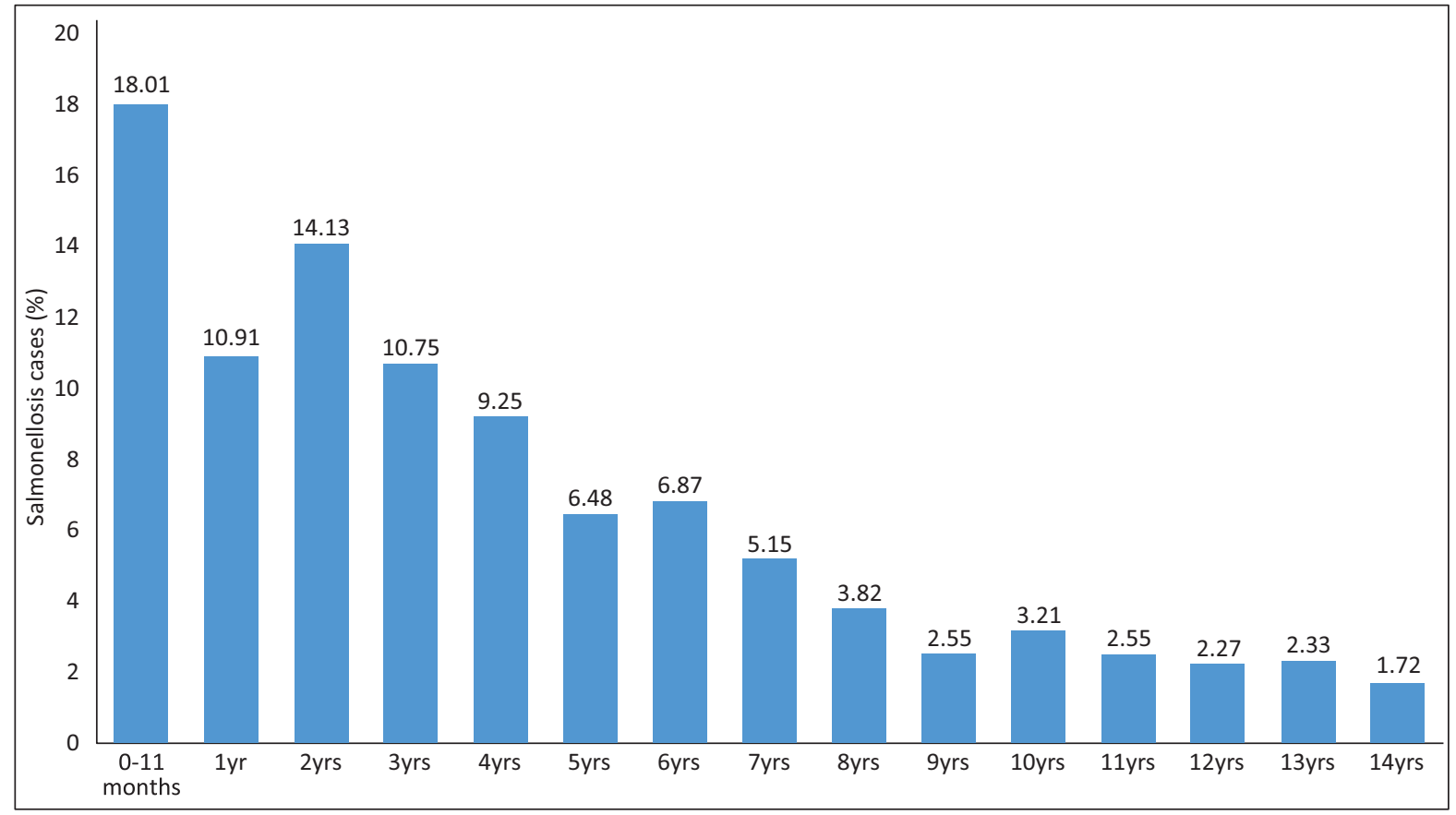

Figure 3. Percentage distribution of Salmonellosis cases in children by age, Greece, 2011-2017.

\section{Serovars and Antimicrobial Resistance}

According to serovar distribution, shown in Figure 4 , only 27 cases (1.1\%) were positively identified as typhoid salmonellosis (12 cases of Salmonella Typhi, $44.4 \%$, and 15 cases of Salmonella Paratyphi B, 55.6\%). Salmonella Enteritidis, Salmonella Typhimurium and Salmonella monophasic Typhimutrim represented 51.2\% in total (Figure 5). Other Salmonella spp. serovars were encountered far less commonly, but accounted for $47.7 \%$ in total
(Figure 5). The increase observed in salmonellosis cases in 2015 and 2016 was predominantly due to an increase in the rates of other Salmonella spp. serovars, while the increase in 2017 was attributed to an increase in both the rates of other Salmonella spp. serovars and the rates of Salmonella monophasic Typhimurium isolates (Figure 5).

The antimicrobial resistance pattern was assessed for 1456 isolates (62\%; 1436 non-typhoid: 20 typhoid). Table 1 presents the resistance of non- 


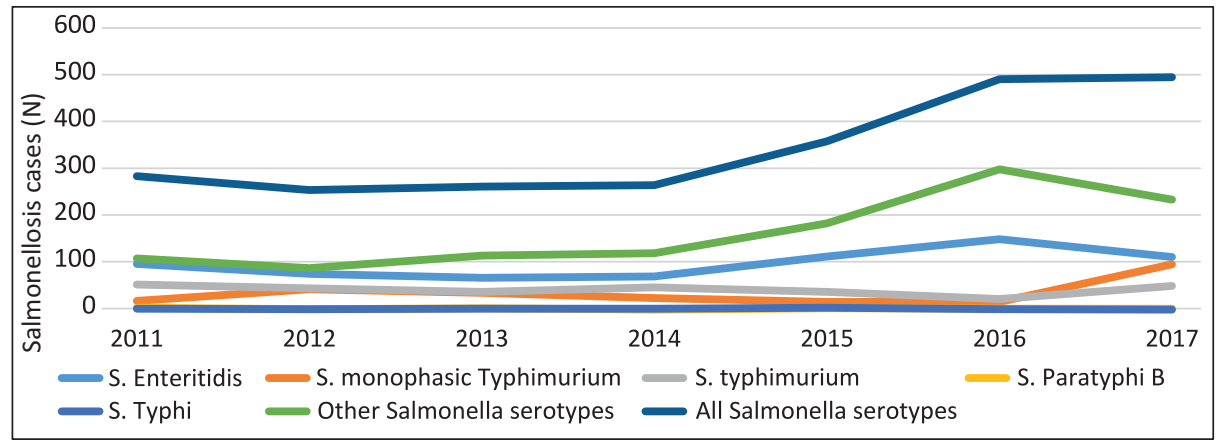

Figure 4. Distribution of Salmonella serovars by year in children $\leq 14$ years old, Greece, 2011-2017.

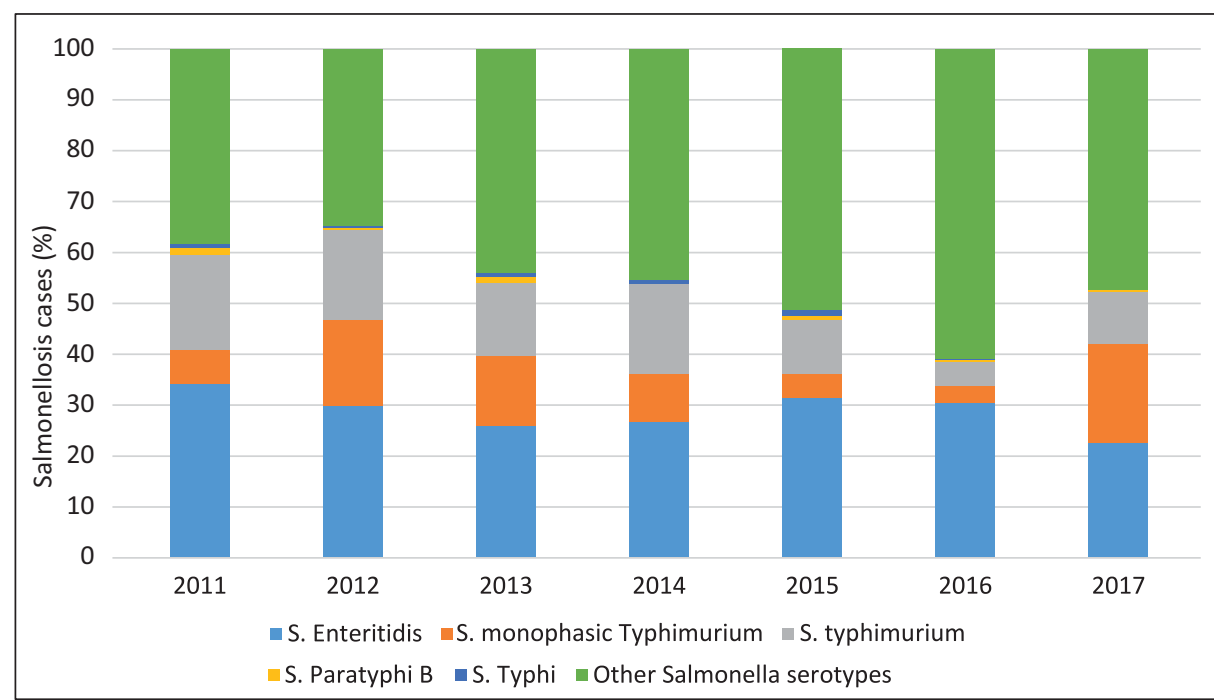

Figure 5. Percentage distribution of Salmonella serovars by year, in children $\leq 14$ years old, Greece, 2011-2017.

typhoid Salmonella spp. isolates during the study period. Resistance to ciprofloxacin, ceftazidime and cefotaxime was low. Resistance to chloramphenicol also remained low (3.6\%). Intermediate rates of resistance were observed against ampicillin and trimethoprim, (17.1\% and $10.6 \%$ respectively). Sulfamethoxazole was associated with the highest resistance rates $(51.4 \%)$, even though it was only available during the sub period of 2014-2017.

Antimicrobial resistance was tested for $414 \mathrm{Sal}$ monella Enteritidis isolates (62\% of the 669 that were identified during the study period). Salmonella Enteritidis exhibited low resistance rates to cefotaxime and ceftazidime $(0.4 \%$ each $)$, while there was no isolate that was resistant to ciprofloxacin. The percentage of the identified MDR isolates also remained low $(6 / 414$ cases, $1.5 \%)$.

Antimicrobial resistance data was assessed for 218 Salmonella Typhimurium isolates $(89 \%$ of the 245 that were totally isolated in the study). All Salmonella Typhimurium isolates were susceptible to ciprofloxacin, while resistance to cefotaxime and ceftazidime was low, $0.5 \%$ and $1 \%$ respectively. Significant resistance rates were recorded to ampicillin, streptomycin, tetracycline and sulfamethoxazole $(49 \%$, 53\%, 55\% and $80 \%$, respectively). A considerable percentage of MDR isolates was also recorded (45/218 cases, $20.6 \%$ ).

Regarding Salmonella monophasic Typhimurium, antimicrobial resistance was tested for 226 Salmonella monophasic Typhimurium isolates out of the 287 cases that were identified (79\%). Salmonella monophasic Typhimurium resistance rates were low for cefotaxime, ceftazidime and ciprofloxacin. $(0 \%, 0.7 \%$, and $0.6 \%$ respectively). However, high rates of resistance were recorded to ampicillin, streptomycin, tetracycline, sulfamethoxazole $(82 \%, 85 \%, 90 \%$ and $89 \%$ respectively). Remarkably, $72.1 \%$ of the isolates during the whole study period were MDR, reaching the percentage of $97.8 \%$ in 2017.

Antimicrobial resistance patterns were available for 20 of the 27 typhoid Salmonella spp. isolates (Table 1). Eight typhoid Salmonella spp. isolates were susceptible to all antibiotics tested. All 
Table 1. Resistance of Salmonella Isolates to the Antimicrobial Agents Tested, among Children $\leq 14$ Years Old, Greece, 2011-2017

\begin{tabular}{|c|c|c|c|c|}
\hline \multirow{2}{*}{ Antimicrobial agent } & \multicolumn{2}{|c|}{ Non-Typhi/Paratyphi isolates } & \multicolumn{2}{|c|}{ Typhi/Paratyphi isolates } \\
\hline & Number & $\%$ & Number ${ }^{*}$ & $\%$ \\
\hline Ampicillin & $245 / 1436$ & 17.1 & $3 / 20$ & 15 \\
\hline Amox/clav ${ }^{+}$ & $21 / 1436$ & 1.5 & $0 / 20$ & 0 \\
\hline Ceftazidime & $15 / 1436$ & 1 & $1 / 20$ & 5 \\
\hline Cefotaxime & $15 / 1436$ & 1 & $1 / 20$ & 5 \\
\hline Amikacin & $5 / 1436$ & 0.3 & $0 / 20$ & 0 \\
\hline Gentamycin & $8 / 719$ & 1.1 & $0 / 20$ & 0 \\
\hline Tobramycin & $69 / 1436$ & 4.8 & $1 / 20$ & 5 \\
\hline Netilmicin & 9/1436 & 0.6 & $0 / 20$ & 0 \\
\hline Streptomycin & $348 / 1436$ & 24.2 & $1 / 20$ & 5 \\
\hline Sulfamethoxazole & $370 / 719$ & 51.4 & $5 / 20$ & 25 \\
\hline Trimethoprim & $152 / 1436$ & 10.6 & $1 / 20$ & 5 \\
\hline Tetracycline & $292 / 1436$ & 20.4 & $1 / 20$ & 5 \\
\hline Chloramphenicol & $52 / 1436$ & 3.6 & $2 / 20$ & 10 \\
\hline Nalidixic acid & $84 / 1436$ & 5,9 & $6 / 20$ & 30 \\
\hline
\end{tabular}

"Number of resistant isolates/Number of total isolates for which the corresponding resistance data was available; †Amoxicillin/Clavulanic Acid.

typhoid Salmonella spp. isolates were susceptible to cefotaxime and ceftazidime, but two Salmonella Paratyphi B isolates that were resistant (10\%). All typhoid Salmonella spp. isolates were susceptible to ciprofloxacin, apart from one Salmonella Typhi isolate that was resistant (5\%). It is noteworthy that typhoid Salmonella isolates were considerably resistant to nalidixic acid and sulfamethoxazole, (30\% and $25 \%$ respectively), whereas they displayed lower resistance profiles to ampicillin and chloramphenicol (15\% and $10 \%$ respectively). Two typhoid Salmonella isolates (one Salmonella Typhi and one Salmonella Paratyphi B) were MDR (10\%).

\section{Bacteremia}

Seventy cases of Salmonella bacteremia were identified during the study period. Nine of these children also yielded Salmonella spp. in their stool cultures (13\%). Out of the twenty-seven different serovars identified in blood, the most common one was Salmonella Enteritidis (10 cases, 14.3\%), followed by Salmonella Oranienburg (8 cases, $11.45 \%$ ) and Salmonella Typhi (6 cases, 8.6\%). The highest rate of Salmonella bacteremia was record- ed in 2017 (14 cases, 20\%). The majority of cases occurred in August, and the lowest in February.

The age distribution of salmonellosis bacteremia cases is shown in Fig. 6. Boys outnumbered girls (ratio 1.8:1). Most cases were recorded in Athens (64.3\%). Antimicrobial resistance was assessed for 41 salmonellosis bacteremia isolates (58.6\%). The highest resistance rates were recorded to sulfamethoxazole (56\%), followed by ampicillin (14.6\%), streptomycin (19.5\%), tetracycline (9.7\%), trimethoprim (9.7\%) and chloramphenicol (7.3\%). Resistance rates were low for the $3^{\text {rd }}$ generation cephalosporins and ciprofloxacin (2.4\% each). Twentyseven Salmonella bacteremia isolates (out of 41 for which antibiotic resistance pattern was available) were susceptible to all antimicrobial agents tested (65.9\%), while 7 isolates were MDR (17\%).

\section{Discussion}

To our knowledge, our study is one of a few reports that have been published so far on salmonellosis epidemiology and antimicrobial resistance rates in children. Salmonellosis rates in children aged $\leq 14$ years old remained almost unchanged during the 
2011-2014 period, albeit they were significantly higher in the following years (2015-2017). A similar trend has been previously reported in salmonellosis rates in all age groups in Greece, most likely attributed to improved hospital staff compliance with reporting procedures, especially following a study highlighting salmonellosis underreporting in Greece compared to other countries $(18,19)$. Alternatively, a recently established weekly comparison of NRCS data with those reported to the HCDCP may have contributed to the increase in salmonellosis rates during the 2015-2017 period. Additionally, this increase was in line with an increase in the number of Salmonella outbreaks in other countries across Europe (19). In detail, on the basis of data of the European Centre for Disease Prevention and Control (ECDC), salmonellosis rates in Europe gradually decreased during the 2007-2013 period. The implementation of an integrated legislative approach by food business operators and policy makers in Europe, to monitor and control salmonellosis along the food chain from primary production to consumption, over the last 10 years thus brought about important progress. Nevertheless, in the years that followed, an increasing trend was observed (19). Alternatively, travel-associated cases may have contributed to this increase. However, Greece was among the countries with the highest proportion of domestic cases versus travel-associated cases, ranging from $86 \%$ to $100 \%$ (19).

In our study, the most commonly identified serovars were Salmonella Enteritidis, Salmonella Typhimurium and Salmonella monophasic Typhimurium. The last is a monophasic variant of Salmonella serovar that lacks the second-phase flagellar antigen encoded by the fljB gene, and its exact antigenic formula is 4,[5],12:i:1,2. Salmonella Enteritidis and Salmonella Typhimurium were also the most common serovars in previous studies conducted in Greece (20-23). Our results agree with those reported by the WHONET, Greek System for the Surveillance of Antimicrobial Resistance. Similar trends have been reported in other European countries (24-26). Low percentages of Salmonella Typhi and Salmonella Paratyphi B were recorded in our study, in accordance with other studies reporting low rates of typhoid Salmonella cases ( 1-2\%) (24-26). The remaining serovars, although quite rarely reported, accounted for nearly half of all reported cases, in agreement with other European reports (24-26). Remarkably, Salmonella monophasic Typhimurium cases increased substantially during the study period, being the second most prevalent serovar in 2017. The first description of a monophasic Salmonella Typhimurium epidemic in Europe was that of a "Spanish clone," in 1997 (24-26). Since then, many European countries have reported the increased incidence of this serovar responsible for foodborne disease, particularly associated with pig herds, but later with cattle $(27,28)$.

The majority of salmonellosis cases were reported among infants, as previously described (29). Remarkably, salmonellosis rates in children were consistently higher compared to adults, since children often present with more severe symptoms and are more frequently admitted (24). Therefore, salmonellosis in children is more likely to be recorded, compared to adults, who most often present with mild symptoms and do not refer to a physician.

The vast majority of salmonellosis cases were reported in Athens, most likely implying better compliance with the reporting procedures. Indeed, city hospitals have easier access to diagnostic procedures compared to regional ones. Continuous monitoring and better staff training in the reporting procedures are required to overcome this problem.

Our results support recently published European data showing low resistance rates to $3^{\text {rd }}$ generation cephalosporins among non-typhoid Salmonella serovars (19). All non-typhoid Salmonella spp. isolates were susceptible to ciprofloxacin, although higher resistance rates have been reported in Europe ( 11\%)(24-26). Resistance rates of non-typhoid Salmonella serovars to chloramphenicol also remained low. Non-typhoid Salmonella spp. isolates displayed considerable resistance rates to sulfamethoxazole, streptomycin, tetracycline and ampicillin, as previously described (24-26). No non-typhoid Salmonella spp. isolate was resistant to ciprofloxacin and cefotaxime. This finding agrees with European data re- 
porting that combined resistance to ciprofloxacin and cefotaxime was $0.6 \%$ (24).

Salmonella Enteritidis isolates displayed low resistance rates to $3^{\text {rd }}$ generation cephalosporins; resistance rates to ciprofloxacin were also low (0\%). Other European data show that Salmonella Enteritidis resistance rates to $3^{\text {rd }}$ generation cephalosporins were also low, whereas a higher proportion of Salmonella Enteritidis isolates (12.3\%) were resistant to ciprofloxacin (24-26). Overall, resistance rates of Salmonella Enteritidis were lower compared to a previous study conducted in Greece (23). Salmonella Typhimurium and monophasic Typhimurium isolates also exhibited low resistance rates to $3^{\text {rd }}$ generation cephalosporins and ciprofloxacin, as previously described in Europe (24-26).

Notably, an increasing prevalence of MDR Salmonella monophasic Typhimurium isolates was observed among children in our study. A similar trend has been previously described in Europe and the USA among the general population. Currently, there are no available data showing that there was also such an increase among the subpopulation of children (30-31).

The antimicrobial resistance profile of typhoid Salmonella isolates in our study is similar to that reported by other European countries (17). Importantly, antimicrobial resistance rates to $3^{\text {rd }}$ generation cephalosporins and ciprofloxacin remain low (19). Significant resistance rates have been reported to ampicillin, chloramphenicol, nalidixic acid and sulfamethoxazole (19). Notably, $10 \%$ of isolates were MDR, while in endemic regions in Sub-Saharan Africa and South-East Asia the corresponding percentage was $>33.3 \%$ (32).

Salmonella bacteremia most commonly affected infants and young children $<5$ years of age, with a slight predominance of boys, similarly to previous reports (22). Salmonella Enteritidis was the most common isolate associated with bacteremia, also frequently isolated in stool cultures, as previously described in Greece (22). Salmonella Typhimurium, Salmonella Enteritidis and Salmonella Dublin are the most frequently related with bacteremia worldwide (33). Antimicrobial resistance rates to clinically important $3^{\text {rd }}$ generation cephalosporins and ciprofloxacin for Salmonella isolates causing bacteremia remained low (34). In our study, almost one in five Salmonella bacteremia isolates (17.1\%) were MDR, similarly to previous studies reporting that MDR isolates are most frequently associated with bacteremia (9-11).

Our study has several limitations, mainly associated with its retrospective design. Firstly, supplementary epidemiological data are often underreported, confounding our results. However, we assume that weekly validation of our data with the HCDCP may have minimized this possibility. Secondly, hospital staff in regional areas (apart from Athens) often do not comply with the reporting procedures, implying that continuous training is required to increase the salmonellosis reporting rates. Besides, most comparisons have been made with studies conducted among adults. Although Salmonella serovars that cause disease in adults and children do not differ significantly, underlying conditions or previous antibiotic use in adults may affect antimicrobial resistance patterns. Furthermore, large amounts of data on epidemiological characteristics and antimicrobial resistance were missing. To overcome this problem, we mentioned the precise number of cases for which the corresponding data were available. Besides, this study was based on data recorded by the NRCS over the period 2011 to 2017. Apart from epidemiological data i.e age, gender, specimen source, geographical data and recent travel abroad, other data regarding the clinical course are not routinely provided. Therefore, such an analysis was not feasible. The inclusion of sulfamethoxazole data for only 4 of the surveillance years is a notable limitation, but that did not preclude us from unveiling the high rates of antimicrobial resistance to this important antimicrobial during the years that it was available. Finally, resistance rates to azithromycin, that is currently an important clinical choice, were not evaluated in our study.

\section{Conclusion}

In conclusion, we have provided a blueprint of current serovar prevalence and antimicrobial resistance of Salmonella isolates among children 
in Greece. Salmonellosis epidemiology in Greek children is comparable to previously published European data. Antimicrobial resistance rates to $3^{\text {rd }}$ generation cephalosporins and ciprofloxacin for non-typhoid and typhoid-paratyphoid remain low. Notably, there is an increasing prevalence of Salmonella monophasic Typhimurium isolates, associated with multiple antimicrobial resistance. Hence, continuous surveillance is mandatory, to monitor such trends.

\section{What Is Already Known on this Topic:}

Salmonellosis is a major health problem worldwide. Resistance to the current 1st line antibiotics, such as ciprofloxacin and 3rd-generation cephalosporins, the higher rates of Salmonella spp. isolates resistant to fluoroquinolones, as well as the emergence of ESBL and MDR clinical isolates are important clinical concerns. This problem has become even worse among children who predominantly present with severe salmonellosis. However, hardly any studies exist describing Salmonella epidemiology and antimicrobial resistance among this vulnerable population.

\section{What this Study Adds:}

This study presents the epidemiological data, serovar distribution and antimicrobial resistance patterns of Salmonella spp. isolates among children aged $\leq 14$ years old in Greece. This information allowed a comparison between Salmonella surveillance data in this population and European data among adults already published, encouraging clinicians to feel comfortable prescribing 3 rd generation cephalosporins and fluoroquinolones as the 1st line empirical treatment of salmonellosis in children. This approach, together with control measures (i.e. avoiding unnecessary antibiotic therapy) will lead to the more judicious use of antimicrobials for the treatment of salmonellosis, with the potential to suppress antimicrobial resistance further with continuous surveillance of salmonellosis epidemiology and antimicrobial resistance.

Authors' Contributions: Conception and design: GG and GM; Acquisition, analysis and interpretation of data: GG and GM; Drafting the article TL; Revising it critically for important intellectual content: GG and GM; Approved final version of the manuscript: GG, TL and GM.

Conflict of Interest: The authors declare that they have no conflict of interest.

\section{References}

1. ECDC E. The European Union summary report on antimicrobial resistance in zoonotic and indicator bacteria from humans, animals and food in 2016. EFSA J. 2018;16(2):5182. DOI: 10.2903/j.efsa.2018.5182

2. Crump JA, Mintz ED. Global trends in typhoid and paratyphoid fever. Clin Infect Dis. 2010;50(2):241-6.

3. Bula-Rudas FJ, Rathore MH, Maraqa NF. Salmonella Infections in Childhood. Adv Pediatr. 2015;62(1):29-58.
4. O'Brien SJ. The "decline and fall" of nontyphoidal salmonella in the United Kingdom. Clin Infect Dis. 2013;56(5):705-10.

5. European Food Safety Authority and European Centre for Disease Prevention and Control (EFSA and ECDC). The European Union One Health 2018 Zoonoses Report. EFSA J. 2019;17(12):e05926.

6. Crump JA, Sjölund-Karlsson M, Gordon MA, Parrye CM. Epidemiology, Clinical Presentation, Laboratory Diagnosis, Antimicrobial Resistance, and Antimicrobial Management of Invasive Salmonella Infections. Clin Microbiol Rev. 2015;28(4):901-37.

7. Chen HM, Wang Y, Su LH, Chiu CH. Nontyphoid Salmonella infection: Microbiology, clinical features, and antimicrobial therapy. Pediatr Neonatol. 2013;54(3):147-52.

8. Kagambèga A, Lienemann T, Aulu L, Traoré AS, Barro N, Siitonen A HK. Prevalence and characterization of Salmonella enterica from the feces of cattle, poultry, swine and hedgehogs in Burkina Faso and their comparison to human Salmonella isolate. BMC Microbiol. 2013;13:253.

9. Hassing RJ, Goessens WHF, van Pelt W, Mevius DJ, Stricker BH, Molhoek N, et al. Salmonella subtypes with increased MICs for azithromycin in travelers returned to the Netherlands. Emerg Infect Dis. 2014;20(4):705-8.

10. Abgottspon $H$, Zurfluh $K$, Nüesch-Inderbinen $M$, Hächler H, Stephan R. Quinolone resistance mechanisms in salmonella enterica serovars hadar, kentucky, virchow, schwarzengrund, and 4,5,12:i:-, isolated from humans in switzerland, and identification of a novel qnrd variant, qnrd2, in s. hadar. Antimicrob Agents Chemother. 2014;58(6):3560-3.

11. Doublet B, Praud K, Nguyen-Ho-Bao T, Argudín MA, Bertrand $S$, Butaye $P$, et al. Extended-spectrum $\beta$-lactamaseand ampc $\beta$-lactamase-producing $\mathrm{D}$-tartrate-positive Salmonella enterica serovar Paratyphi B from broilers and human patients in Belgium, 2008-10. J Antimicrob Chemother. 2014;69(5):1257-64.

12. Arnoldini M, Vizcarra IA, Pena-Miller R, Stocker N, Diard M, Vogel V, et al. Bistable expression of virulence genes in Salmonella leads to the formation of an antibiotic-tolerant subpopulation. PLoS Biol. 2014;12:e1001928.

13. Claudi B, Sprote P, Chirkova A, Personnic N, Zankl J, Schurmann N, et al. Phenotypic variation of Salmonella in host tissues delays eradication by antimicrobial chemotherapy. Cell. 2014;158(4):722-33.

14. Wirth T. Massive lineage replacements and cryptic outbreaks of Salmonella Typhi in eastern and southern Africa. Nat Genet. 2015;47(6):565-7.

15. Colobatiu L, Tabaran A, Flonta M, Oniga O, Mirel S, Mihaiu M. First description of plasmid-mediated quinolone resistance determinants and beta-lactamase encoding genes in non-typhoidal Salmonella isolated from humans, one companion animal and food in Romania. Gut Pathog. 2015;7:16. doi:10.1186/s13099-015-0063-3. 
16. Wong VK, Baker S, Pickard DJ, Parkhill J, Page AJ, Feasey NA, et al. Phylogeographical analysis of the dominant multidrug-resistant H58 clade of Salmonella Typhi identifies inter- and intracontinental transmission events. Nat Genet. 2015;47(6):632-9.

17. European Centre for Disease Prevention and Control. EU Protocol for Harmonized Monitoring of Antimicrobial Resistance in Human Salmonella and Campylobacter Isolates - June 2016. Stockholm: ECDC; 2016.

18. Mellou K, Saranti-Papasaranti E, Mandilara G, Georgakopoulou T. Laboratory capacity of Greek hospitals for diagnosis of salmonellosis and surveillance systems' performance in the years of economic crisis, 2010-2016. Epidemiol Infect. 2019;147:e17.

19. European Food Safety Authority. The European Union summary report on trends and sources of zoonoses, zoonotic agents and food-borne outbreaks in 2017. EFSA Journal. 2018;16(12):5500.

20. Maraki S, Papadakis IS. Serotypes and antimicrobial resistance of human nontyphoidal isolates of salmonella enterica from crete, Greece. Interdiscip Perspect Infect Dis. 2014;2014:256181.

21. Maraki S, Mantadakis E, Nioti E, Katragkou A, Samonis G. Serotype distribution and antibiotic resistance of human gastrointestinal isolates of salmonella enterica from Crete, Greece. J Chemother. 2009;21(2):222-5.

22. Papaevangelou V, Syriopoulou V, Charissiadou A, Pangalis A, Mostrou G, Theodoridou M. Salmonella bacteraemia in a tertiary children's hospital. Scand J Infect Dis. 2004;36(8):547.

23. Tassios PT, Markogiannakis A, Vatopoulos AC, Katsanikou E, Velonakis EN, Kourea-Kremastinou J, et al. Molecular epidemiology of antibiotic resistance of Salmonella enteritidis during a 7-year period in Greece. J Clin Microbiol. 1997;35(6):1316-21.

24. European Centre for Disease Prevention and Control. Annual Epidemiological Report 2016 - Typhoid and paratyphoid fever. [Internet]. Stockholm: ECDC; 2016.
25. Collard JM, Bertrand S, Dierick K, Godard C, Wildemauwe C, Vermeersch K, et al. Drastic decrease of Salmonella Enteritidis isolated from humans in Belgium in 2005, shift in phage types and influence on foodborne outbreaks. Epidemiol Infect. 2008;136(6):771-81.

26. ECDC. Disease Data from ECDC Surveillance Atlas -Salmonellosis.; 2018. Available from: https://www.ecdc.europa.eu/en/salmonellosis/surveillance-and-disease/atlas.

27. Animal Health and Veterinary Laboratories Agency. Department for Environment and Food and Rural Affairs. Salmonella in Livestock Production in Great Britain, 2013. Surrey: Animal Health and Veterinary Laboratories Agency; 2013.

28. Moreno Switt AI, Soyer Y, Warnick LD, Wiedmann M. Emergence, distribution, and molecular and phenotypic characteristics of Salmonella enterica serotype 4,5,12:i:-. Foodborne Pathog Dis. 2009;6(4):407-15.

29. Sinha A, Sazawal S, Kumar R, Sood S, Reddaiah VP, Singh B, et al. Typhoid fever in children aged less than 5 years. Lancet. 1999;354(9180):734-7.

30. Petrovska L, Mather AE, Abuoun M, Branchu P, Harris SR, Connor T, et al. Microevolution of monophasic Salmonella typhimurium during epidemic, United Kingdom, 2005-2010. Emerg Infect Dis. 2016;22(4):617-24.

31. Shippy DC, Bearson BL, Holman DB, Brunelle BW, Allen HK, Bearson SMD. Porcine Response to a Multidrug-Resistant Salmonella enterica serovar I 4,[5],12:i:- Outbreak Isolate. Foodborne Pathog Dis. 2018;15(5):253-61.

32. Bhan MK, Bahl R BS. Typhoid and Paratyphoid Fever. Lancet. 2005;366(9487):749-62.

33. Ao TT, Feasey NA, Gordon MA, Keddy KH, Angulo FJ, Crump JA. Global Burden of Invasive Nontyphoidal Salmonella Disease, 2010. Emerg Infect Dis. 2015;21(6):941-9.

34. Crump JA, Medalla FM, Joyce KW, Krueger AL, Hoekstra RM, Whichard JM, et al. Antimicrobial resistance among invasive nontyphoidal Salmonella enterica isolates in the United States: National Antimicrobial Resistance Monitoring System, 1996 to 2007. Antimicrob Agents Chemother. 2011;55(3):1148-54. 